\title{
Application of the qSOFA score to predict mortality in patients with suspected infection in a resource-limited setting in Malawi
}

\author{
Michaëla A. M. Huson ${ }^{1,2,3}$ - Chawezi Katete ${ }^{3}$ Lilian Chunda ${ }^{3} \cdot$ Jonathan Ngoma $^{3}$ • \\ Claudia Wallrauch $^{3}$ - Tom Heller ${ }^{3,4}$ Tom van der Poll ${ }^{1}$ - Martin P. Grobusch ${ }^{1,2}$
}

Received: 1 June 2017 / Accepted: 31 July 2017 / Published online: 7 August 2017

(c) The Author(s) 2017. This article is an open access publication

\begin{abstract}
Purpose To determine the predictive value of qSOFA (quick Sequential Organ Failure Assessment) in Malawian patients with suspected infection.

Methods Prospective observational study in a tertiary referral hospital in Malawi.

Results Predictive ability of qSOFA was reasonable [AUROC 0.73 (95\% CI 0.68-0.78)], increasing to 0.77 (95\% CI 0.72-0.82) when classifying all patients with altered mental status as high risk. Adding HIV status as a variable to the qSOFA score did not improve predictive value.

Conclusion qSOFA is a simple tool that can aid risk stratification in resource-limited settings.
\end{abstract}

Keywords Sepsis - qSOFA · Resource-limited setting · HIV

Electronic supplementary material The online version of this article (doi:10.1007/s15010-017-1057-5) contains supplementary material, which is available to authorized users.

Michaëla A. M. Huson

m.a.huson@amc.uva.nl

1 Division of Infectious Diseases, Center of Experimental and Molecular Medicine, Academic Medical Center,

University of Amsterdam, Meibergdreef 9, Room G2-105, 1105 AZ Amsterdam, The Netherlands

2 Division of Infectious Diseases, Center of Tropical Medicine and Travel Medicine, Academic Medical Center, University of Amsterdam, Amsterdam, The Netherlands

3 Medical Department, Kamuzu Central Hospital, Lilongwe, Malawi

4 Lighthouse HIV Clinic, Kamuzu Central Hospital, Lilongwe, Malawi

\section{Introduction}

The Third International Consensus Definitions for Sepsis and Septic Shock proposes the use of a simplified SOFA (Sequential Organ Failure Assessment) score, termed quickSOFA (qSOFA), for patients with suspected infection outside intensive care to rapidly identify those at high risk for dying [1,2]. The qSOFA score includes respiratory rate, altered mental status, and systolic blood pressure which are readily available in any setting. As such, the qSOFA score could be of particular relevance in resource-limited regions. We previously did a retrospective analysis of the qSOFA score in adult patients admitted to hospital in Lambaréné, Gabon, with suspected infection (including bacterial infection and/or malaria). In this setting, a qSOFA score $\geq 2$ had a sensitivity of $87 \%$ (95\% CI $60-98 \%$ ) and specificity of $75 \%$ (95\% CI 70-80\%), with an area under the receiver-operating characteristic (AUROC) of 0.83 (95\% CI 0.74-0.93) in patients with suspected infection [3]. We report here on the first prospective qSOFA score study in a resource-limited setting. We examined the performance of qSOFA to predict mortality in patients with suspected infection in Kamuzu Central Hospital (KCH), a 750-bed tertiary care hospital in Lilongwe, Malawi, with a catchment population of over 5 million people. A previous study in this hospital demonstrated that altered mental state, which is also included in qSOFA, was associated with high mortality rates [4], but the qSOFA score has not been previously evaluated in $\mathrm{KCH}$.

\section{Methods}

\section{Data collection}

During 6 weeks in November and December 2016, all consecutive patients admitted to the adult medical ward of $\mathrm{KCH}$ 
were included. The qSOFA score was calculated for all with suspected infection, defined by the start of antibiotics, antimalarials or antituberculous medication. The qSOFA score allocates one point for each of the following: respiratory rate $\geq 22 /$ min, altered mentation [Glasgow Coma Scale (GCS) of $\leq 14$ ], or systolic blood pressure $\leq 100 \mathrm{mmHg}$. Data collection was based on routine clinical observations, supplemented by a dedicated physician who checked all new admissions twice daily and recorded vital signs included in the qSOFA score in case of missing data. Therefore, every patient had a qSOFA score recorded within $12 \mathrm{~h}$ of admission. HIV status and final diagnoses were recorded, based on routine clinical observations, as well as in-hospital mortality (the primary study endpoint), and duration of hospital stay. Patients who were transferred out or who absconded were excluded from the analysis. All data were entered into an anonymized excel database. Informed consent need was waived by the ethical board of $\mathrm{KCH}$.

\section{Statistical analyses}

Continuous variables are presented as median with interquartile range [IQR]. Categorical variables are expressed as number and percentage. Comparisons were made using a Mann-Whitney $U$ test for continuous variables and Fisher's exact $t$ test for categorical variables. Patients with missing values for the qSOFA score were excluded from the primary analyses. To assess the performances of the qSOFA to predict in-hospital mortality, we calculated sensitivity, specificity, and negative and positive predictive values for a qSOFA score of $\geq 2$. We also calculated the area under the receiver-operating characteristic curve (AUROC). All statistical analyses were 2 -tailed, and a $P$ value $<0.05$ was considered significant. GraphPad Prism was used for statistical analyses (GraphPad Software, La Jolla, California).

\section{Results}

During the study period 650 patients were admitted, of which $518(80 \%)$ had a suspected infection. Information on follow-up or qSOFA score was incomplete in 60 patients, leaving 458 patients for analysis. Baseline characteristics are given in Table 1. In-hospital mortality was $23 \%$ and $41 \%$ of patients had a qSOFA score $\geq 2$, reflecting the very sick population that $\mathrm{KCH}$ receives as a tertiary referral center. Mortality was $3 \%$ in patients with a qSOFA score of 0 , and $13 \%, 35 \%$ and $63 \%$ in patients with a qSOFA score of 1,2 , and 3, respectively. A HIV test result was available for $87 \%$ of patients, of whom 54\% was positive. The most common diagnosis was suspected bacterial infection. The qSOFA score outperformed GCS as a single parameter to predict mortality, with an AUROC of 0.73 (95\% CI 0.68-0.78) compared to 0.70 (95\% CI 0.63-0.76; Table 2). However, sensitivity was increased from $72 \%$ (95\% CI 62-80) to $79 \%$ (95\% CI 70-87) by allocating more weight to the GCS in the qSOFA score, thus classifying both patients with qSOFA $\geq 2$ or GCS $<15$ as a high-risk group, while specificity decreased only moderately from $68 \%$ (95\% CI 63-73) to $63 \%$ (95\% CI 58-68), resulting in an AUROC of 0.77 (95\% CI 0.72-0.82).

A large patient proportion was HIV positive; so we also assessed the impact of adding HIV status as an additional risk factor to the qSOFA score. By allotting one extra point for HIV seropositivity but still applying a $\geq 2$ cut-off to the total score, sensitivity increased to $87 \%$ (78-93) (Table 2). However, specificity decreased to $44 \%(38-50)$ and the AUROC fell to $0.68(0.62-0.74)$. We also analyzed the performance of the qSOFA score separately in HIV positive and HIV negative patients (Supplementary Table 1 and 2) and found predictive value to be equal in both groups.

\section{Discussion}

We found a reasonably good ability of the qSOFA score to predict mortality with an AUROC of 0.73 (95\% CI $0.68-0.78$ ), which could be increased to 0.77 (95\% CI $0.72-0.82$ ) when patients with altered mental status were classified as high risk regardless of other qSOFA criteria.

The qSOFA score performance was less in the study reported here compared to our retrospective study in Gabon, where we found an AUROC of 0.83 (95\% CI 0.74-0.93). Possibly, the differences are related to severity of illness on presentation, or standards of care. As such, there may not be a one-size-fits-all risk score. qSOFA may serve as a simple tool for risk stratification, but requires further prospective validation in different settings. For example, others have compared qSOFA to the CURB-65 for pneumonia patients and found improved predictive ability of the qSOFA score when age of $\geq 65$ or greater was added as risk factor [5]. The value of such an addition depends highly on the patient population, as the median age of our patients was 35 years, with only $11 \%$ of patients aged above 64 . We also examined the added value of HIV status in risk stratification; however, in the Malawian setting, this resulted in a very low specificity, leading to misclassification of low-risk patients. Prioritizing limited resources to such a large group of patients is unsustainable, so we do not recommend incorporating HIV status in risk stratification in our setting.

Limitations of our study include the single-center design and lack of a definite diagnosis in most patients. However, this reflects the clinical reality in many resource-limited 
Table 1 Baseline characteristics of study participants

\begin{tabular}{|c|c|c|c|c|}
\hline Characteristics & All patients $n=458$ & In-hospital death $n=106$ & Discharged $n=352$ & $P$ value \\
\hline \multicolumn{5}{|l|}{ Demographics } \\
\hline Male, $n(\%)$ & $243(53.1)$ & $63(59.4)$ & $180(51.1)$ & 0.15 \\
\hline Age (years), median [IQR] & $35[26-47]$ & $40[30-52]$ & $33.5[25-45]$ & 0.001 \\
\hline \multicolumn{5}{|l|}{ HIV status } \\
\hline HIV status known, $n(\%)$ & $397(86.7)$ & $92(86.8)$ & $305(86.6)$ & 1.0 \\
\hline HIV positive, $n(\%)^{\mathrm{a}}$ & $213(53.7)$ & $50(54.3)$ & $163(53.4)$ & 0.91 \\
\hline On cART, $n(\%)^{\mathrm{b}}$ & $166(77.9)$ & $39(78)$ & $127(77.9)$ & 1.0 \\
\hline New HIV diagnosis, $n(\%)^{\mathrm{b}}$ & $20(9.4)$ & $4(8)$ & $16(9.8)$ & 1.0 \\
\hline \multicolumn{5}{|l|}{ qSOFA parameters } \\
\hline BP systolic (mmHg), median [IQR] & $110[97-122]$ & $110[91.5-129]$ & $111[98-121]$ & 0.37 \\
\hline Respiratory rate (breaths/min), median [IQR] & $28[20-32]$ & $28[24-37.75]$ & $27[20-32]$ & 0.002 \\
\hline Altered mental status, $n(\%)$ & $117(25.5)$ & $59(55.7)$ & $58(16.5)$ & $<0.0001$ \\
\hline qSOFA $0, n(\%)$ & $69(15.1)$ & $2(1.9)$ & $67(19)$ & $<0.0001$ \\
\hline qSOFA $1, n(\%)$ & $201(43.9)$ & $28(26.4)$ & $173(49.1)$ & $<0.0001$ \\
\hline qSOFA $2, n(\%)$ & $169(36.9)$ & $64(60.4)$ & $105(29.8)$ & $<0.0001$ \\
\hline qSOFA $3, n(\%)$ & $19(4.1)$ & $12(11.3)$ & $7(2)$ & 0.0002 \\
\hline $\mathrm{qSOFA} \geq 2, n(\%)$ & $188(41)$ & $76(71.7)$ & $112(31.8)$ & $<0.0001$ \\
\hline \multicolumn{5}{|l|}{ Diagnoses } \\
\hline Suspected bacterial infection ${ }^{c}$ & $225(49.1)$ & $63(59.4)$ & $162(46)$ & 0.012 \\
\hline Probable bacterial infection ${ }^{\mathrm{d}}$ & $37(8.1)$ & $5(4.7)$ & $32(9.1)$ & 0.22 \\
\hline РTB & $30(6.6)$ & $6(5.7)$ & $24(6.8)$ & 0.82 \\
\hline ЕРТВ & $40(8.7)$ & $12(11.3)$ & $28(8)$ & 0.33 \\
\hline Cryptococcal meningitis & $9(2)$ & $1(0.9)$ & $8(2.3)$ & 0.31 \\
\hline Malaria & 35 (7.6) & $3(2.8)$ & $32(9.1)$ & 0.04 \\
\hline Gastro-enteritis & $38(8.3)$ & $3(2.8)$ & $35(9.9)$ & 0.02 \\
\hline cART failure & $12(2.6)$ & $1(0.9)$ & $11(3.1)$ & 0.31 \\
\hline Other & $14(3.1)$ & $5(4.7)$ & $9(2.6)$ & 0.33 \\
\hline \multicolumn{5}{|l|}{ Secondary outcome } \\
\hline Length of stay (days), median [IQR] & $4[2-7]$ & $2.5[1-5]$ & $5[2-7]$ & $<0.0001$ \\
\hline
\end{tabular}

$I Q R$ inter quartile range, $c A R T$ combination antiretroviral therapy, $q S O F A$ quick sequential organ failure assessment, $B P$ blood pressure, $P T B$ pulmonary tuberculosis, EPTB extrapulmonary tuberculosis

${ }^{a}$ Percentage of patients with a known HIV status

${ }^{\mathrm{b}}$ Percentage of HIV positive patients

${ }^{c}$ Defined by the initiation of antibiotic therapy

${ }^{\mathrm{d}}$ Diagnosis supported by imaging or culture results

Table 2 Predictive value of qSOFA

\begin{tabular}{lllll}
\hline & qSOFA $\geq 2$ & GCS $<15$ & $\begin{array}{l}\text { qSOFA } \geq 2 \text { and/or } \\
\text { GCS }<15\end{array}$ & qSOFA + HIV status $\geq 2$ \\
\hline Sensitivity, \% (95\% CI) & $72(62-80)$ & $56(46-65)$ & $79(70-87)$ & $87(78-93)$ \\
Specificity, \% (95\% CI) & $68(63-73)$ & $84(79-87)$ & $63(58-68)$ & $44(38-50)$ \\
Positive predictive value, \% (95\% CI) & $40(33-47)$ & $50(41-60)$ & $39(33-46)$ & $32(26-38)$ \\
Negative predictive value, \% (95\% CI) & $89(84-92)$ & $86(82-90)$ & $91(86-94)$ & $92(86-95)$ \\
AUROC (95\% CI) & $0.73(0.68-0.78)$ & $0.70(0.63-0.76)$ & $0.77(0.72-0.82)$ & $0.68(0.62-0.74)$ \\
\hline
\end{tabular}

qSOFA quick sequential organ failure assessment, GCS Glasgow coma scale, AUROC area under the receiver-operating characteristic 
settings and a risk stratification score should be useful regardless of the final diagnosis at discharge.

In conclusion, we report the first prospective data on the ability of qSOFA to predict mortality in a resource-limited setting and found that the qSOFA score is a simple tool that can aid risk stratification in this environment. However, the predictive performance of qSOFA may vary between patient populations. Our observations were made in a very sick population in a tertiary referral centre, and we emphasize the need to further validate qSOFA in different settings, for example, in primary care in resource-limited areas, where qSOFA might help select patients for referral.

Acknowledgements We thank all patients for their participation and all doctors and nursing staff of Kamuzu Central Hospital for their hard work and support of this study.

\section{Compliance with ethical standards}

Conflicts of interest On behalf of all authors, the corresponding author states that there is no conflict of interest.

Open Access This article is distributed under the terms of the Creative Commons Attribution 4.0 International License (http://creativecommons.org/licenses/by/4.0/), which permits unrestricted use, distribution, and reproduction in any medium, provided you give appropriate credit to the original author(s) and the source, provide a link to the Creative Commons license, and indicate if changes were made.

\section{References}

1. Singer M, Deutschman CS, Seymour CW, Shankar-Hari M, Annane D, Bauer M, et al. The third international consensus definitions for sepsis and septic shock (sepsis-3). JAMA. 2016;315:801-10. doi:10.1001/jama.2016.0287.

2. Seymour CW, Liu VX, Iwashyna TJ, Brunkhorst FM, Rea TD, Scherag A, et al. Assessment of clinical criteria for sepsis: for the third international consensus definitions for sepsis and septic shock (sepsis-3). JAMA. 2016;315:762-74. doi:10.1001/ jama.2016.0288.

3. Huson MA, Kalkman R, Grobusch MP, van der Poll T. Predictive value of the qSOFA score in patients with suspected infection in a resource limited setting in Gabon. Travel Med Infect Dis. 2016;15:76-77. doi:10.1016/j.tmaid.2016.10.014.

4. Harrington B, Kyriakos Vorkas C, Kanyama C, Ngoma J, Hoffman I, Hosseinipour MC. Altered mental status is an indicator of mortality and associated with both infectious and non-communicable disease in Lilongwe, Malawi. Trop Dr. 2015;45:164-7. doi:10.1177/0049475515577024.

5. Kolditz M, Scherag A, Rohde G, Ewig S, Welte T, Pletz M, et al. Comparison of the qSOFA and CRB-65 for risk prediction in patients with community-acquired pneumonia. Intensive Care Med. 2016;42:2108-10. doi:10.1007/s00134-016-4517-y. 